\title{
DEVELOPMENT OF 2-DOF ADAPTIVE MECHATRONIC DEVICE WITH CORRECTIVE ADJUSTMENT OF LASER TRACKER REFLECTOR FOR INDUSTRIAL ROBOT CALIBRATION
}

\author{
Dmitrii Kosterev, Andrei Vorotnikov, Simeon Nedelchev, \\ Elena Romash \& Yuri Poduraev
}
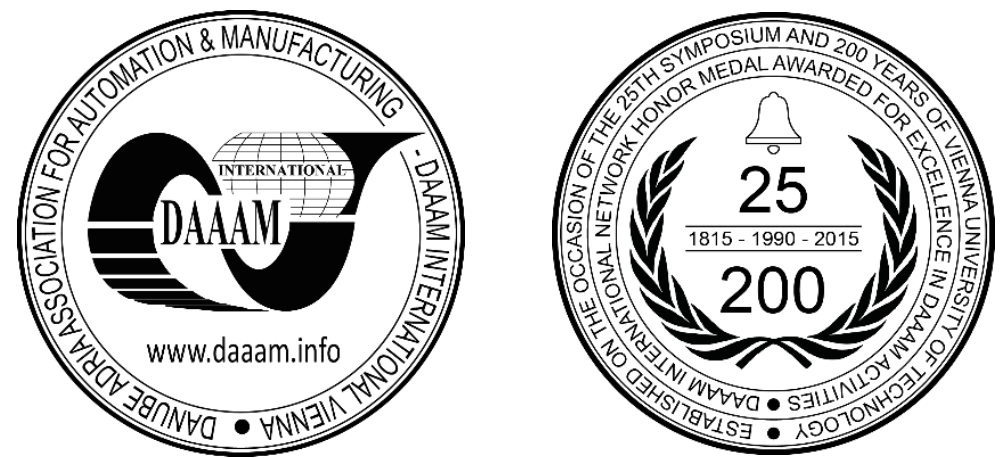

This Publication has to be referred as: Kosterev, D[mitrii]; Vorotnikov, A[ndrei]; Nedelcev, S[imeon]; Romash, E[lena] \& Poduraev, Y[uriy] V[.] (2017). Development of 2-DOF Adaptive Mechatronic Device with corrective adjustment of laser tracker reflector for Industrial Robot Calibration, Proceedings of the 28th DAAAM International Symposium, pp.1010-1014, B. Katalinic (Ed.), Published by DAAAM International, ISBN 978-3-902734-11-2, ISSN 1726-9679, Vienna, Austria

DOI: $10.2507 / 28$ th.daaam.proceedings.140

\begin{abstract}
In this paper, a design of adaptive mechatronic device for industrial robot calibration was proposed. An adaptive mechatronic module can be used to permanently preserve the orientation of the reflector with respect to the source of measuring tool. A testing setupfcor based on coordinate measuring machine - laser tracker LTD 800 was developed. The calibration method of one degree of freedom of proposed device was described and experimentally evaluated.
\end{abstract}

Keywords: industrial robot calibration; laser tracker; measurement; mechatronics; control system

\section{Introduction}

Currently, industrial robots are actively used in all areas of production. They perform a large number of different tasks, such as palletizing, welding, painting, assembling or metalworking. Each operation can be performed with an appropriate quality, which is provided by the performance characteristics of the robot. In accordance with the standard ISO 9283 [1], one of the main characteristics of an industrial robot is the pose accuracy, path accuracy, the path velocity accuracy and repeatability. Achievement of these characteristics is provided in two ways: the appropriate calculation at the design stage, and calibration, which is necessary as a procedure after the assembly of the manipulator. If the robot has a good repeatability, which can only be provided by design, calibration can be used to achieve the corresponding performance in the remaining characteristics. Thus, calibration is necessary to improve the performance and accuracy of an industrial robot.

To carry out the calibration procedure, a software approach is used, in which the parameters of the control model are calibrated. Parameter calibration involves 2 steps: measuring of the necessary positions or trajectories of the robot end effector depending on the calibrated characteristic, and processing of the obtained data. After estimation of parameters, it is necessary to perform validation of the calibration state, which allows to guarantee correctness of the procedure. 
There are different schemes for measuring the position and orientation of end effector of the robot during calibration, different metrological equipment is used [2]. Thus, the researchers in [3] calibrate the robot using cameras. Robertson, Slocum and Willoughby [4] shows a specialized precision tooling with goniometers. Approach based on using virtual linear guidance for calibration presented in [5]. Slamani, Nubiola and Bonev [6] used the Renishaw XL-80 and Ball Bar systems to calibrate robots.

All the presented measurement approaches have a limitations on the possible range of the measured robot positions, therefore in this article the measurement of the positions or trajectories of the robot required for calibration and validation is carried out using a coordinate measuring machine - the laser tracker shown in Fig. 1. For the measurements, the reflector of the laser tracker is mounted on the robot flange. The laser tracker measures the coordinates of the reflector's during the movement of the robot. Unfortunately, the reflector of the laser tracker has a limited laser reception area and a high cost. In order to cover full robot work space, some researchers use at least eight expensive reflectors [7]. Leica laser trackers [8], provide solution of this problem, based on optical principles. However, practically the entire range of the working area can be measured using a specialized equipment - 2-DOF mechatronic module, presented in the patent API Radian [9]. But, unfortunately, the rotation angles in the joints of this module are related to the robot control system, which makes the user's work more difficult. Therefore, it is advisable to develop an adaptive mechatronic module that has its own control system capable of making decisions without a high-level control system, hence the connection between the mechatronic module and the robot control system will be absent. Such a control system can be built on the inertial sensors.

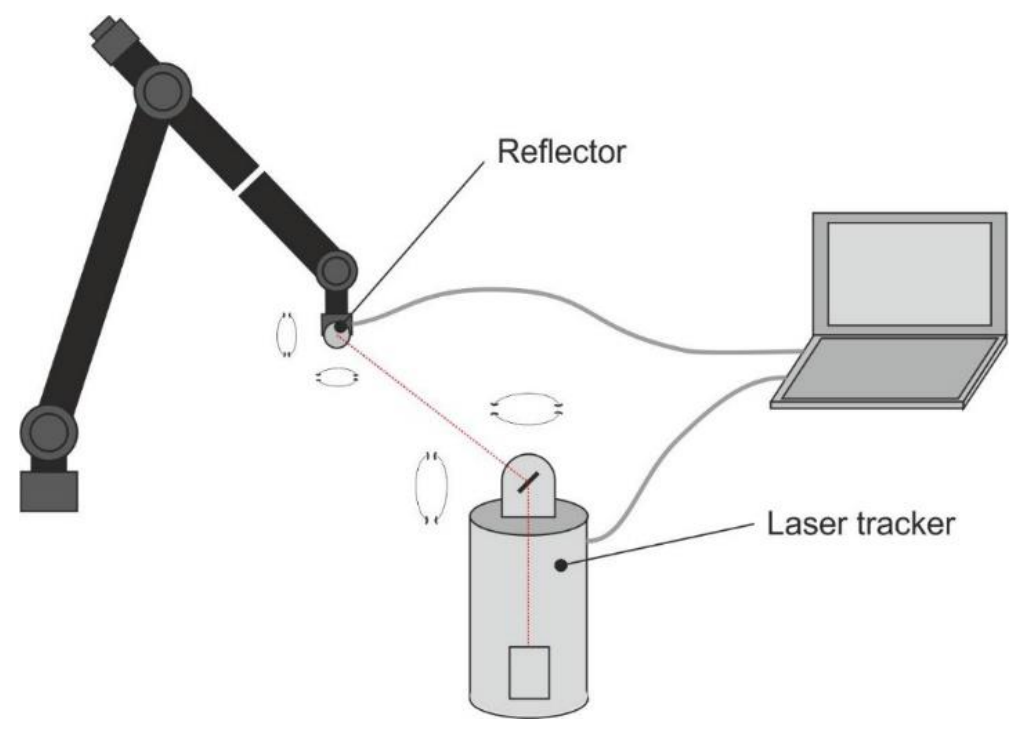

Fig. 1. Scheme of measurements of the manipulator calibration using a laser tracker.

This paper is aimed at describing the concept of 2-DOF adaptive mechatronic module that adjusts to the beam of a laser tracker regardless of the position of the industrial robot and experimental evaluation of 1-DOF of proposed module. A single adaptive mechatronic module will allow to obtain the coordinates of the positions and trajectories of the industrial robot necessary for calibration and validation procedures without additional manual rearrangement of the reflectors. Proposed system will help to maintain constant orientation of the reflector relative to the laser tracker during movement of robot, which leads to significant reducing a time required for calibration and validation of industrial robots. Since proposed module also required calibration, we perform experimental

\section{The concept of a 2-DOF adaptive mechatronic module}

The appearance of the 2-DOF adaptive mechatronic module used to calibrate industrial robots is shown in Figure 2.b. Its kinematic diagram, shown in Figure 2.a, is designed so that the reflector mounted on the adaptive mechatronic module is in the center intersection of the axes of the mechatronic module being developed. The direct kinematics problem was solved with using Denavit-Hetenberg approach [10], position and orientation of the reflector in space are calculated depending on the given angles of motors shafts. Coordinate systems $\mathrm{X}_{1} \mathrm{Y}_{1} \mathrm{Z}_{1}$ (first axis) $\mathrm{X}_{2} \mathrm{Y}_{2} \mathrm{Z}_{2}$ (second axis), are shown in Figure 2.a. Matrices of homogeneous transformations of the transition between local coordinate systems module include the following parameters: $\Theta_{i}-$ angle that characterizes the amount of rotation of the $X_{1}$ around the $Z_{1}$ axis. $n$ the adaptive mechatronic module, $\Theta_{1}$ is the generalized coordinate for the first joint; $d_{i}-$ displacement between $X_{1}$ and $X_{2}$ along the mutual perpendicular along $Z_{1} ; a_{i}$ - the shortest distance between the beginning of the first coordinate system and the 2-nd coordinate system measured along the $X_{2}$ axis; $\alpha_{i}$-angle, on which it is necessary to turn the axis $Z_{1}$ around the axis $X_{2}$, so that it becomes co-oriented with the axis $Z_{2}$. Each of the parameters defines a matrix of homogeneous transformations. The product of these matrices is a homogeneous matrix of the Denavite-Hetenberg transformations. 


$$
A_{(i-1, i)}=A_{(i-1, i)}^{d} A_{(i-1, i)}^{\theta} A_{(i-1, i)}^{a} A_{(i-1, i)}^{\alpha}=\left(\begin{array}{cccc}
\cos \theta i & -\sin \theta i \cos \alpha i & \sin \theta i \sin \alpha i & a i \cos \theta i \\
\sin \theta i & \cos \theta i \cos \alpha i & -\cos \theta i \sin \alpha i & a i \sin \theta i \\
0 & \sin \alpha i & \cos \alpha i & d i \\
0 & 0 & 0 & 1
\end{array}\right)
$$

Thus, the complete kinematic model of the mechatronic module can be represented by the following relation:

$$
H=\left(\begin{array}{cccc}
\cos \theta_{1} & -\cos \alpha_{1} \sin \theta_{1} & \sin \alpha_{1} \sin \theta_{1} & a_{1} \cos \theta_{1} \\
\sin \theta_{1} & \cos \alpha_{1} \cos \theta_{1} & -\sin \alpha_{1} \cos \theta_{1} & a_{1} \sin \theta_{1} \\
0 & \sin \alpha_{1} & \cos \alpha_{1} & d_{1} \\
0 & 0 & 0 & 1
\end{array}\right) \cdot\left(\begin{array}{cccc}
\cos \theta_{2} & -\sin \theta_{2} & 0 & 0 \\
\sin \theta_{2} & \cos \theta_{2} & 0 & 0 \\
0 & 0 & 1 & 0 \\
0 & 0 & 0 & 1
\end{array}\right)
$$

Where, $\mathrm{H}$ - the resulting matrix of position and orientation of the adaptive mechatronic module. Using this matrix, the current orientation of the reflector is calculated, depending on the value of the motors shafts angles. A significant advantage over similar systems is the absence of mechanical transmission, which reduce the cost, improve controllability, accuracy and simplicity of system.

a)

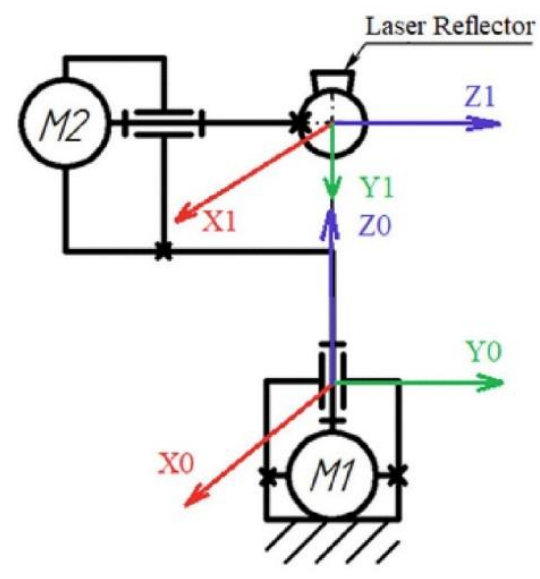

b)

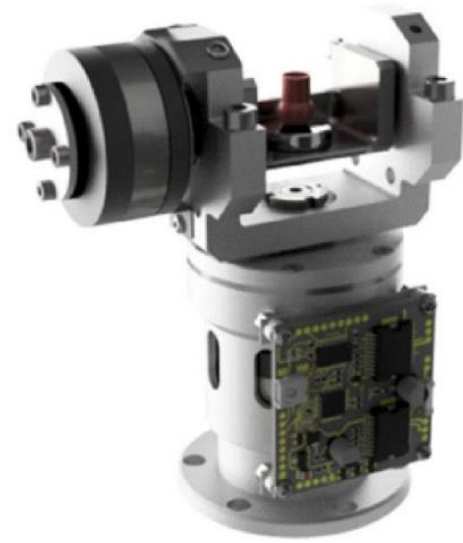

Fig. 2. a) Kinematic diagram of the mechatronic module with local coordinate systems, b) Appearance of mechatronic module.

Proposed module able to make decisions about the necessary change of the motor shaft angles preserve the orientation of the reflector, relative to the tracker beam source. Information about the current orientation of the reflector comes from the inertial sensors (accelerometer, gyro, compass). The data enters the BDPT microcontroller and, after filtering by the Kalman algorithm, converted into Euler orientation angles. Thus, inertial sensors operate as feedback elements of the mechatronic module servo drives.

The implementation of all the above solutions, should provide the accuracy of the reflector positioning in a sphere with a diameter of $15 \mu \mathrm{m}$, and the error in orientation of the reflector within an angle of 8 degrees; however, all this is achievable with precision manufacturing. For an affordable implementation, without using of high-precision CNCs, the concept of a specialized trimmer is proposed. It is able to adjust the reflector of the laser tracker to the point of intersection of the two axes of the mechatronic module. This is achieved by adding to the reflector 4 microscrews with micromotors, capable of alternately leading the reflector to the intersection point shown in Figure 3.

\section{Experimental results.}

In order to ensure the accurate operation of the adaptive mechatronic module, it is necessary to calibrate it. As it was described in previous section, it is necessary to perform a multi-stage adjustment, which will ensure the rotation of the reflector around the intersection point of the module axes. The main two parts of this procedure are the adjustment of the center of the reflector to the axes of rotation of the mechatronic module, first to the first axis, then to the second axis. The adjustment operation to the actual position of each axis of the mechatronic module includes a set of identical actions: the rotation of the reflector around the tuned axis and its linear displacement. And linear movement is carried out by means of two micro-screws. 
To confirm the hypothesis of the possibility of adjusting the reflector to the actual position of one axis of rotation, an experimental setup was developed and an appropriate experiment was carried out. The experimental setup shown in Figure 3. consists of the following set of elements:

2-DOF micro stage (1) with 2 micrometer screws (2) Is mounted on the output shaft of the stepping motor with the aid of a rig (3), made on a 3D printer. A magnetic base (4) is mounted on the micro stage, which, in turn, is fitted with a reflector (5) of the laser tracker (6) Leica LTD800. The motor (7) is rigidly fixed by means of a vice (8). The control of the stepper motor is realized with the help of a controller (9) and a driver (10) of the stepper motor, which are powered by $12 \mathrm{~V}$ from a laboratory power source (11).

The elements and control system of the rig are located on the antivibration table (12). The motor shaft rotates with the reflector, while the laser tracker measures its position. With full rotation of the motor shaft and measurement of the position of the laser tracker, a circle with a center lying on the axis of rotation of the engine is formed.

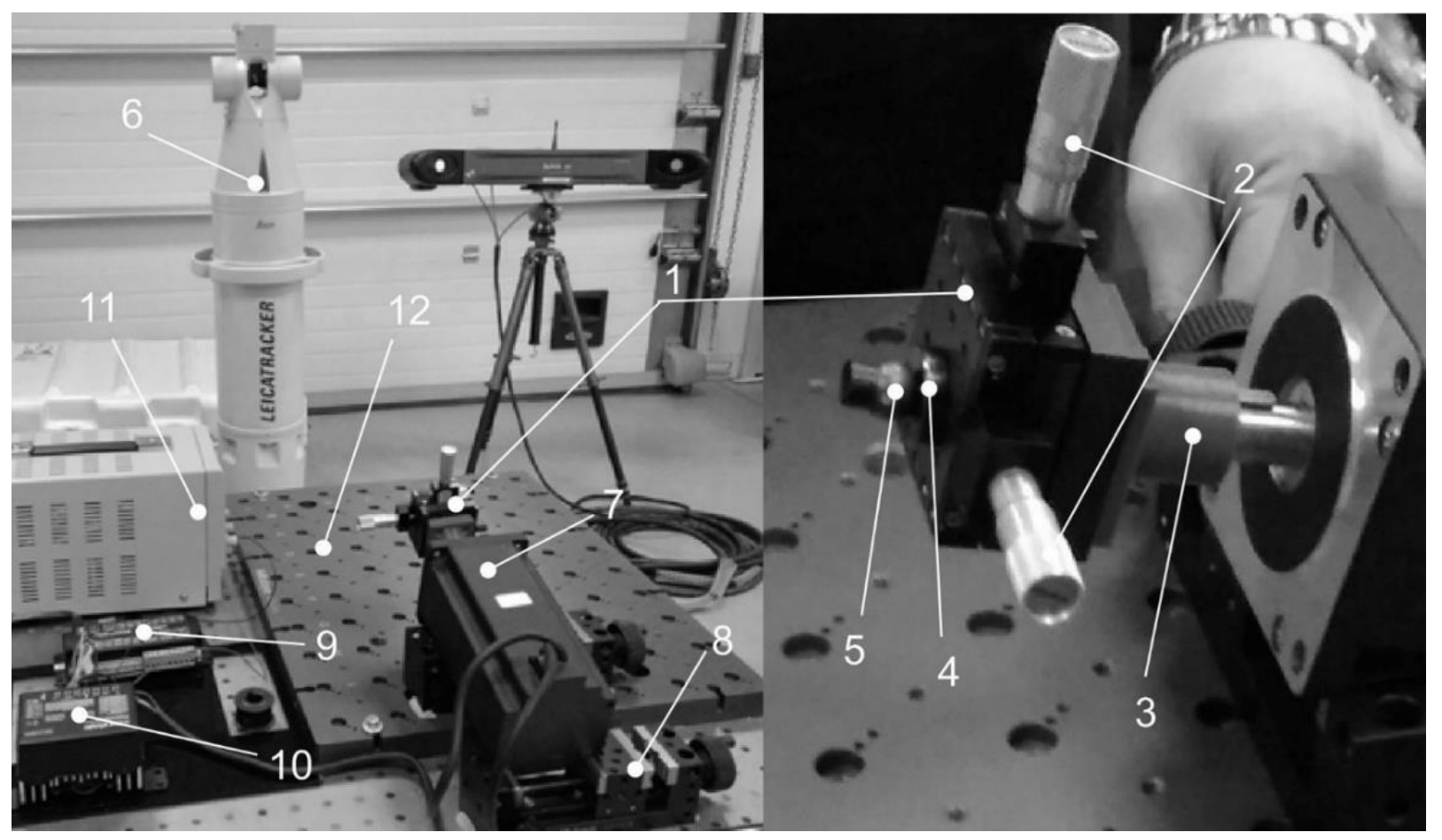

Fig. 3. Experimental setup.

The experiment for adjusting the reflector contained 7 iterations, each of which is a $360^{\circ}$ rotation of the motor shaft. With each complete rotation, the reflector position was measured by a laser tracker. The measurements were carried out in dynamic mode. After receiving the data points $\left(x_{i}, y_{i}, z_{i}\right)$ from each iteration, the circle center coordinates $\left(x_{0}, y_{0}\right)$ which lies on the motor shaft axis, were calculated. Further, one of the micro-screws is used, which moves the magnetic base together with the reflector closer to the center of the circle.

The next iteration involves another micro-screw. And, thus, in turn, the reflector moves to the axis of rotation of the motor shaft. Data processing is performed using the methods proposed in [11-13], in order to solve the following optimization:

$$
\begin{aligned}
& \sum_{i=1}^{n}\left(A x_{i}+B y_{i}+C z_{i}+1\right)^{2} \rightarrow \min \\
& \sum_{i=1}^{n}\left[\left(x_{i}-x_{0}\right)^{2}+\left(y_{i}-y_{0}\right)^{2}-R_{0}{ }^{2}\right]^{2} \rightarrow \min
\end{aligned}
$$

Where $(A, B, C)$ - parameters of norm vector of plane where lies points $\left(x_{i}, y_{i}, z_{i}\right), R_{0}$ - radius of reflector in each iteration.

The data obtained from each rotation is illustrated in figure 4.a. The first 6 iterations give circles, using which it is necessary to determine the centers of circles. The last iteration gives a set of points with a maximum spread of $13 \mu \mathrm{m}$, and these are extreme points that are outside the range of $6 \sigma$ of the normal distribution. $2 \sigma$ make up a little more than 4 $\mu \mathrm{m}$. A graph illustrating the decrease in the circle radius with respect to the iteration number is shown on the figure 4.b. 


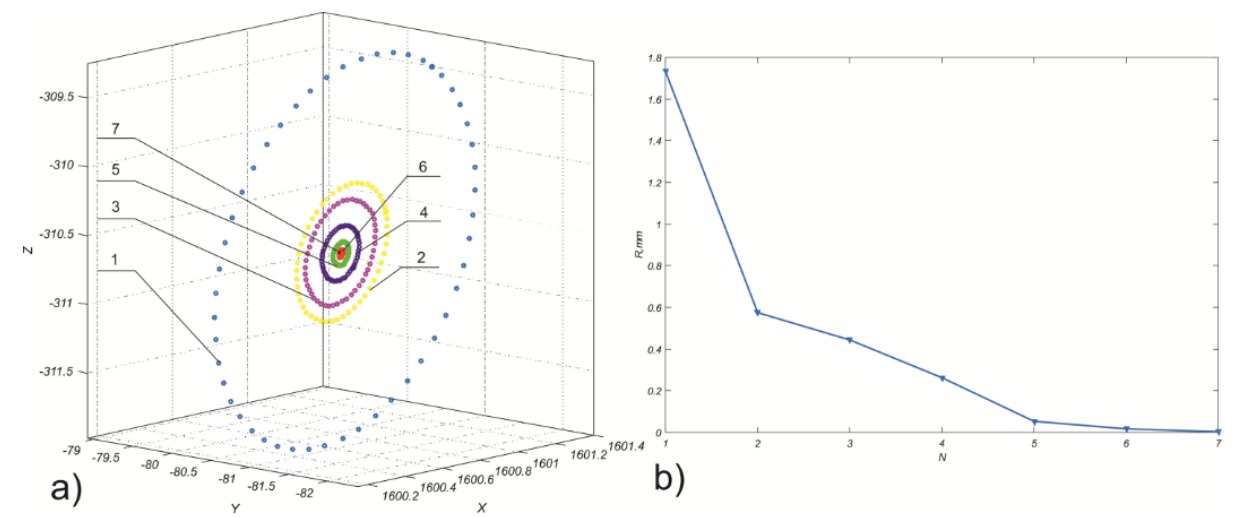

Fig. 4. a) A visual display of the data obtained, b) A decrease in the radius of the circle as the iteration of the corrective adjustment is increased.

Experiment showed that procedure described above let us to reduce error between center of the motor shaft and reflector from $1.8 \mathrm{~mm}$ to $13 \mu \mathrm{m}$ after 7 iterations.

\section{Conclusion}

In this paper concept of 2-DOF adaptive mechatronic device has been proposed. Following device allow to improve volume of working space in which we can measure position of industrial robot without manual rearrangement of the reflectors. The control system of the adaptive mechatronic module will able to make decisions without a industrial robot control system. With using the proposed adaptive mechatronic module, the time of procedures for calibrating and validating mechanisms will be significantly reduced. The use of a specialized system for the installation of reflectors will reduce the requirements for the accuracy of the components of the adaptive mechatronic module, which was experimentally approved thru calibration of 1-DOF stepper motor based system. The maximum projected error of position determination on the basis of the performed experiments and after the appropriate calculation does not exceed $15 \mu \mathrm{m}$.

The next step will be to conduct an experiment for 2-DOF and prepare for the production of the device prototype, debug the control system and software to interact with the computer.

\section{References}

[1] Manipulating Industrial robots (1995). - Performance criteria and related test methods, ISO 9283:1998.

[2] Manipulating industrial robots (1998). - Informative guide on test equipment and metrology methods of operation for robot performance evaluation in accordance with ISO 9283, ISO/13309:1995.

[3] Petar Maric and Velibor Djalic, (2011). Choice of window size in calibrating the geometry of manipulators based on the regions correlation, ELECTRONICS, VOL. 15, NO. 1, JUNE 2011, pp.45-53.

[4] Robertson, A., Slocum, A., Willoughby, P. (2001). "Precision robot calibration using kinematically Placed inclinometers." Proceedings of 2001 ASPE Conference, p 229 - 232.

[5] Biqiang Du, Ning Xi, Erick Nieves, (2012). Industrial robot calibration using a virtual linear constraint, International Journal On Smart Sensing And Intelligent Systems, Vol. 5, No. 4, December 2012, pp. 987-1001.

[6] Mohamed Slamani, Albert Nubiola and Ilian Bonev, (2012). Assessment of the positioning performance of an industrial robot, Industrial Robot: An International Journal, 39/1, 2012, pp. 57-68, DOI 10.1108/01439911211192501.

[7] Albert Nubiola, Ilian A. Bonev, (2013). Absolute calibration of an ABB IRB 1600 robot using a laser traker, Robotics and Computer_Integrated Manufacturing, 2013, Vol. 29, pp. 236-245.

[8] Isolde Dressler, (2012). Modeling and control of stiff robots for flexible manufacturing, Department of Automatic Control Lund University Lund, Printed in Sweden by Media-Tryck, 2012.

[9] Kam C. Lau, Yuanqun Liu, Guixiu Qiao, Liangyun Xie, (2010). "Volumetric error compensation system with laser tracker and active target,” U.S. Patent US20100176270 A1, July 15, 2010.

[10] Denavit J., Hartenberg R. (1955). A kinematic notation for lower-pair mechanisms based on matrices. // Transactions of ASME - Journal of Applied Mechanics, 22(2), pp. 215-221, June 1955.

[11] Mooring B., Roth Z., Driels M. Fundamentals of manipulator calibration// John Wiley \& Sons, INC, 1991.

[12] Vorotnikov A., Bashevskaya. O., Ilyukhin Yu., Romash, E., Isaev A.V., Poduraev Yu. (2016). Geometrical Approach for Industrial Robot Axis Calibration Using Laser Tracker // Proceedings of the 26th DAAAM International Symposium. - Vienna, Austria, 2016. - P. 0897-0904

[13] Vorotnikov A[ndrei]; Romash E[lena]; Isaev,A[lexander]; Bashevskaya O[lga]; Bianchi G[iacomo] \& Poduraev Y[uri] (2016). Uncertainty estimation of axes direction determination of industrial robot using an ellipsoid concentration model, Proceedings of the 27th DAAAM International Symposium, pp.0480-0486, B. Katalinic (Ed.), Published by DAAAM International, ISBN 978-3-902734-08-2, ISSN 1726-9679, Vienna 